\title{
Bloggers and Readers Blogging Together: Collaborative Co-creation of Political Blogs
}

\author{
Eric P. S. Baumer ${ }^{3}$, Mark Sueyoshi ${ }^{2} \&$ Bill Tomlinson ${ }^{1}$ \\ ${ }^{1}$ Donald Bren School of Information and Computer Sciences, Department of Informatics, University \\ of California, 5029 Bren Hall, Irvine, CA 92697-3440, USA; ${ }^{2}$ International Studies / East Asian \\ Cultures, University of California, Irvine, CA, USA; ${ }^{3}$ Information Science Department, Cornell \\ University, 301 College Ave, Ithaca, NY 14850, USA (Phone: +1-949-8249333; Fax: +1-949-8244056; \\ E-mail: ericpsb@cornell.edu)
}

\begin{abstract}
A significant amount of research has focused on blogs, bloggers, and blogging. However, relatively little work has examined blog readers, their interactions with bloggers, or their impact on blogging. This paper presents a qualitative study focusing specifically on readers of political blogs to develop a better understanding of readers' interactions with blogs and bloggers. This is the first such study to examine the same blogging activity from both readers' and bloggers' perspectives. Readers' significance and contributions to blogs are examined through a number of themes, including: community membership and participation; the relationship between political ideology, reading habits, and political participation; and differences and similarities between mainstream media (MSM) and blogs. Based on these analyses, this paper argues that blogging is not only a social activity, but is a collaborative process of co-creation in which both bloggers and readers engage. Implications of this finding contribute to the study and understanding of reader participation, to the design of technologies for bloggers and blog readers, and to the development of theoretical understandings of social media.
\end{abstract}

Key words: blog readers, blogging, blogs, online activism, political blogs, social media

\section{Introduction}

Social media, and blogs in particular, have at times been described as enabling the democratization of production (Blood 2004; McKenna and Pole 2004). Rather than a few large corporations or publishing houses producing all available content, virtually anyone with an internet connection can become a producer. These media can take many forms-blogs, Flickr streams, YouTube videos, Twitter feeds-but all share the commonality of making content production available to anyone in possession of the necessary technologies. This paper is concerned specifically with practices surrounding one form of social media: blogging. While most previous research has focused on bloggers, e.g., (Nardi et al. 2004b; Reed 2005; Lenhart 2005), or used techniques such as social network analysis on blogs (Adamic and Glance 2005), relatively little attention has been 
paid to readers of blogs (Baumer et al. 2008). This paper seeks to take a more comprehensive approach, examining the processes through which both bloggers and readers engage in the collaborative co-creation of blogs.

Specifically, we explore these processes of collaborative co-creation in the context of political blogs. Blogging has become a significant political force (Wallsten 2005; McKenna and Pole 2004; Adamic and Glance 2005), not only in terms of influencing discourse in mainstream media (MSM) (Wallsten 2005; Drezner and Farrell 2004), but also in terms of its capacity to democratize and encourage participation in political discussion (McKenna and Pole 2004; Walker 2006). Studying the reading of political blogs is an important component to understanding how that force takes effect. This paper presents a study of readers of political blogs.

This focus on political blogs was motivated by a number of factors. First, this paper seeks to expand upon previous research on blog readers. In a previous study examining the practice of blog reading in general, Baumer et al. (2008) suggest that, due to the variety of blogging practices they found, it is important to follow up their study with research aimed at specific types of blogging, such as political blogs, which is the focus of this paper. Second, while Baumer et al. focus specifically on reading behaviors, this paper attempts to understand better the ways in which bloggers and readers interact. The study presented here is, to the authors' knowledge, the first that examines the same blogging activity through the experiences of both bloggers and readers. Other previous research has argued that political blogs serve as a public forum for engaging in political discussion and debate (Wallsten 2005; McKenna and Pole 2004; Walker 2006). We seek to develop a deeper understanding of these social and political processes by exploring them through the experiences of individual bloggers and readers. Looking at such discussion and debate from both these perspectives provides an opportunity to study more closely the interactions between the two, and to consider the extent to which blog reading might be considered a form of political participation. These various attributes of political blogs provide an opportunity to study this paper's central theme: the collaborative co-creation of blogs by bloggers and readers.

Undertaking such a study, however, requires critically examining some of the analytic distinctions commonly applied to blogging. For instance, the distinction between bloggers and readers implies a producer/consumer relationship. Baumer et al. point to the fuzziness of this distinction, arguing that "it might be better to consider the question in terms of degree of membership, where an individual may be both a blogger and a reader to varying and independent degrees" (Baumer et al. 2008, p.1112). Here, we suggest an alternative possibility: rather than trying to determine if a given individual is a reader or a blogger, perhaps we might consider instead the extent to which a given activity may be reading oriented, writing oriented, or both. We are not arguing that a distinction between bloggers and readers cannot be made or that such a distinction is never useful. Indeed, this 
paper at times refers to an individual as a blogger or as a reader when such analytic categories help make clearer the different parts played in co-creating the blog. Rather, we wish to problematize the blogger/reader binarism, suggesting, as mentioned above, that making such a clear cut distinction can obfuscate the socially distributed nature of the blogging activity. An analysis that focuses strictly on bloggers, or strictly on readers, does not capture the interactions between them and, thus, does not provide a comprehensive picture of blogging.

The primary contribution of this paper, then, is an examination and explication of the various ways in which bloggers and readers engage in collaborative cocreation using analysis and examples from a qualitative study of political blogging. Section 2 reviews related work dealing with lurkers (2.1) and political blogging (2.2). Section 3 describes our study methodology, including recruitment, data collection, and analysis procedures, as well as a profile of our participants. Findings are presented in Section 4, organized around the themes of community, membership, and participation (4.1); the relationships between political ideology, reading habits, and political participation (4.2); and readers' perceptions of mainstream media and social media (4.3). Section 5 weaves together these analytic threads, arguing that each elucidates aspects of the ways in which bloggers and readers participate in the activity of blogging together and presenting a variety of implications of this argument. Section 6 suggests some areas for future work, and Section 7 summarizes the paper's main contributions and implications.

\section{Related Work}

There has been a great deal of research conducted around the topic of blogging. For a summary of the varied and increasingly large literature on blogs, see Schmidt (2007). Little work, however, has focused specifically on readers of blogs. One recent study (Farrell et al. 2008) represents the first analysis of largescale, quantitative data about blog readers, focusing on those who read political blogs. While Farrell, Lawrence, and Sides examine a few specific questions about political deliberation and political participation using quantitative data gathered from many readers of blogs, the study presented here examines the subjective, lived experience of reading political blogs, focusing specifically on the ways in which both bloggers and readers contribute to the creation of the blog. The most directly relevant work is a previous study exploring the habits and practices of blog reading (Baumer et al. 2008). Drawing on an interactional perspective, that study focused on the ways in which people interact with blogs as a way of understanding the social practice of blog reading. This paper focuses more closely on the interactions between readers and bloggers and the ways in which both share in the blogging process, specifically in the context of political blogs. 


\subsection{Lurkers}

Reading blogs can in some ways be compared to lurking on forums or discussion lists (DLs) (Nonnecke and Preece 2001; Preece et al. 2004). A lurker is "someone who has never posted in the community to which s/he belongs" (Preece et al. 2004, p.202). Nonnecke and Preece's work presents a number of themes and findings that are useful in the present discussion. For example, lurkers do not always lurk; the same individual may only lurk in some communities but be an active contributor in others. Similar patterns are described, here and elsewhere (Baumer et al. 2008), about readers who comment actively on some blogs but never on others.

However, while lurkers and blog readers share a number of common practices and behaviors, the activity of blog reading differs from lurking in a number of important ways. First, the technology of blogs makes a clear distinction on any given blog who the blogger is and who the readers are; only bloggers can create a new post, bloggers have far more control over formatting and layout, and bloggers can delete comments or ban specific commenters. A DL without contributions is not a discussion; if everyone lurks, there is no discussion to be had. On the other hand, while many bloggers encourage comments, not all do. In fact, for a blog, it may be more important to have a large number of readers, even readers who do not comment, than to have only a few readers who comment regularly. Many DLs require registration in order to contribute, some even requiring registration in order to view them, while most blogs can be read casually or infrequently without the need of an explicit action of joining. Subscribing to a blog's RSS feed in some ways resembles joining a DL, but the difference is that RSS subscription is not a prerequisite for reading the blog, whereas joining the DL is often a prerequisite for following the discussion. Galegher et al. refer to "the permeable boundaries of electronic groups" (1998, p.524). While they argue that Usenet groups, which resemble DLs in many respects, have permeable boundaries, the blogs' less explicit and less common requirement to join or subscribe make their boundaries somewhat more permeable. Furthermore, blogging can be seen partially as a performative act of self-presentation (boyd 2002; Reed 2005; Lenhart 2005). In such a context, the blogger needs an audience for which to perform, but may not necessarily need that audience to interact. While DLs also provide an environment for online presentation-of-self, this medium by its definition requires interaction in a way that blogging, while it invites explicit interaction, does not necessitate it. This paper compares a number of findings presented here to those dealing with lurkers.

\subsection{Politics Online and Political Blogs}

Some previous work has looked at the uses of online communication by political campaigns (Foot and Schneider 2006). This work focused primarily on official 
sites maintained by candidates rather than independent efforts or blogging specifically. There has also been some discussion of the use of blogs in political campaigns (Carpenter et al. 2004). Previous work has also examined individuals' choices about reading political content that agrees or disagrees with their personal views (Garrett 2005). Results indicate a pattern of selective exposure, where individuals seek out news sources that resonate with their views much more frequently, but they do not often expressly avoid sources with conflicting views.

Several previous studies have focused specifically on political blogs. An analysis of post citations and page link counts of left- and right-leaning blogs (Adamic and Glance 2005) found a "cyberbalkanization" of political blogs in terms of inter-linking and citations. The division between the left and right-leaning blogs is further emphasized by the sources cited, the political figures discussed, and even the political cartoons used. In comparing blogs to MSM, Wallsten (2005) argues that blogs sometimes act as an echo chamber by covering the same material as MSM. This echo chamber effect is most pronounced for certain issues, particularly those relating to foreign policy, such as the war in Iraq, and moral values, such as stem cell research. Furthermore, the degree of the effect varies along ideological lines, with Republican blogs echoing MSM about different topics than Democratic blogs. While not focused specifically on political blogs, Gilbert et al. (2009) analyze agreement and disagreement in blog comments from a variety of genres; they include five major political blogs in their analysis. Comments in their data set were coded as agree, disagree, or neither, based on the commenter's response to the post on which the comment was made. Of the comments on political blogs, $40 \%$ were neither, $47 \%$ agreed, and 13\% disagreed. Gilbert et al. argue that these results support the conclusion that blogging is an echo chamber, in that far more comments agree with a post than disagree.

An important feature distinguishing the work of Adamic and Glance's (2005) and Gilbert et al. (2009) from that of Wallsten (2005) is the set of blogs from which their data are derived. Adamic and Glance (2005) focus specifically on "A-list" blogs, using the top 20 liberal blogs and the top 20 conservative blogs, in terms of how many times the blog is linked to; Gilbert et al.'s (2009) sample includes blogs with the highest Technorati authority ranking. In contrast, Wallsten focuses specifically on non-A-list, "ordinary" political blogs "by the rest of us" (cf. Schiano et al. 2004) to examine how "ordinary citizens are increasingly using political blogs as a form of political expression and - possibly—a form of political participation" (Wallsten 2005, p.10).

McKenna and Pole (2004) assert that political blogging does indeed constitute a form of political participation, which they define as "people talking and acting in the public realm with the aim of coming to agreement about community affairs" (McKenna and Pole 2004, p.9), explicitly including not only voting but also campaigning, contributing to candidates, and contacting representatives. Their results indicate that political bloggers see their blogging as a form of participation, and that they feel they have a significant influence through blogging. Wallsten 
(2005), on the other hand, questions whether or not blogging constitutes political participation, which he defines, informed by (Rosenstone and Hansen 1993), as "action directed explicitly toward influencing the distribution of social goods and social values." Emphasizing the importance of the individual's motive for blogging, Wallsten asserts that political blogs are not so much about political participation as political expression. "If ... bloggers are using their blogs as ... technological 'soapboxes'," rather than to "encourage their readers to sign on-line petitions or mobilize their readers to vote," then political blogging does not constitute political participation. There is the possibility that a blog might simultaneously be motivated by political expression and political participation, but Wallsten does not discuss this possibility, and the empirical focus of his study does not address bloggers' motivations. There is a resonance between the concept of political participation (Wallsten 2005; McKenna and Pole 2004) and the notion of "being a part" of a blog (Baumer et al. 2008), which is explored in more detail in the findings section below.

Importantly, very little of the previous literature on political blogging have focused specifically on readers of political blogs. While McKenna and Pole (2004) cite statistics about the numbers of comments received by their subjects (A-list bloggers), they do not focus specifically on the readers. Walker (2006) performed a preliminary analysis of comments on several A-list political blogs, arguing that commenting might constitute a new form of political participation. Farrell et al. (2008) perform a large-scale analysis of survey data from readers of political blogs, focusing on polarization and participation. Their data show that an overwhelming majority read blogs that agree with their political ideology and opinions, and also that blog reading is associated with increased political participation in terms of voting, monetary contributions to a candidate, and trying to convince others to vote for a particular candidate. Thus, reading political blogs is associated with both increased polarization and increased participation.

While such previous work helps provide a context in which to situate this paper, no previous studies have sought to understand the experience of reading political blogs or to examine how bloggers and readers work together to shape a blog. How and why do readers contribute to, and participate in, blogging? How does reading a blog affect and interaction with activities off the blog? What is the reader's significance and perception thereof? How do readers interact with bloggers, and how do those interactions shape the blog? In an exploratory study of blog readers, Baumer et al. (2008) begin to address some of these questions. However, they suggest that future studies should focus on how blog reading is practiced and constituted in specific contexts. This paper focuses on one such context: political blogging.

\section{Methods}

This study began with a search for blogs from which to recruit readers. Since the focus here is examining how blogger-reader interactions shape a blog, there were 
two important attributes we used to screen potential blogs: the readers and the bloggers. First, in terms of the readers, we wanted to include blogs with a large enough base of frequent readers to have an active, engaged audience, but not so many readers that there were hundreds of comments on every post. That is, we were not recruiting from A-list blogs (Wallsten 2005), as was the case with the few previous studies related to readers of political blogs (Walker 2006; Farrell et al. 2008). This focus on blogs with a slightly smaller but still interactive readership facilitated examining the ways in which readers take part in shaping the blog. Second, in terms of the blogger, we looked for blogs written primarily by a single individual, ${ }^{1}$ rather than group blogs. Ideally, we hoped to include one liberal, left-leaning blogger and one conservative, right-leaning blogger. We wanted blogs with relatively frequent updates (roughly 3 to 10 times per week) and that evidenced some form of reader interaction, either through comments or, in the case of blogs without comments, direct quoting of contributions from readers by the blogger. In this way, we ensured that the blogs we selected evidenced both readers interacting with bloggers and bloggers interacting with readers. Lastly, we needed to find bloggers who were willing both to post recruitment material for our study on their site and to participate in the study themselves.

To find such blogs, we began with Technorati's list of political blogs, ${ }^{2}$ which lists blogs ordered by Technorati Authority. Authority is calculated based on a combination of linking behavior, content, and other metadata, and it is meant to measure a blog's influence in the blogosphere. Most high-authority blogs were Alist blogs with massive readerships and were thus not well-suited for this study; the Huffington Post, one of the top-rated political blogs, routinely receives several hundred, and sometimes over 1,000, comments on virtually every post. To find blogs with more moderately sized reader bases, we searched through the blogrolls of these A-list blogs, then searched recursively through those blogs' blogrolls until we found a list of potential blogs that fit our requirements. This procedure was conducted beginning with both A-list liberal blogs and A-list conservative blogs to generate a list of potential blogs on both sides of the political spectrum. We started by soliciting one each of the conservative and liberal bloggers. The first liberal blogger we contacted agreed to post recruitment information and participate. The first conservative blogger we contacted did not respond. We then contacted five different conservative bloggers before one agreed to post recruitment information and participate in the study. The study information we asked the bloggers to post included a general description of the research procedures, the goal of understanding "the role that readers play in shaping blogs," and a chance to be entered in a drawing for a $\$ 100$ Amazon gift card.

The study methodology was qualitative and ethnographic, consisting primarily of open-ended, semi-structured interviews with each of twelve participants, including two bloggers and five readers of each of their blogs. Each reader was interviewed at least once, and we conducted follow-up interviews with 
approximately half of the blog readers after an initial round of open coding (see below for more details on the analysis process). This resulted in a total of 16 interviews, each of which lasted approximately 45 to 75 minutes. Interviews consisted of questions about general blog-reading practices, such as how readers choose which blogs to read, when and how often they read them, when and how they decide to comment, and examples of interactions they have had with bloggers through and outside of blogs. We also included politically-oriented questions, such as whether readers were motivated largely by news, commentary, humor, writing style, or other aspects; whether readers chose to read blogs of mostly similar or mostly different political ideology; if their blog reading was linked to political engagement, such as voting, public demonstrations, petitioning, or campaign contributions; and whether they felt that political blogs, and in particular their reading of them, made a significant impact. These interviews produced a rich, complex data set providing a deep understanding of participants' experiences.

After these interviews with readers, we interviewed the blogger from each blog to understand their perspectives on blogging and interaction with readers. The blogger interviews were conducted last so that they could be informed by the responses from the blog reader interviews. This is the first study of which the authors are aware to look at the same blogging activity from both the readers' and the blogger's perspectives. All interviews were conducted over the phone or via Skype, except for one participant; Walter (see the Table 1 below) preferred not to speak on the phone and instead provided extensive responses to a written questionnaire via email.

Interviews were also informed by the content of the blogs. During the study, the researchers conducting the interviews regularly read the two blogs from which participants were recruited. The posts and comments were not included as

Table 1. List of participants; first 10 are readers, last 2 are bloggers.

\begin{tabular}{|c|c|c|c|c|}
\hline Name & Gender & Age & Political ideology & Recruited from \\
\hline Eli & M & - & "left leaning" & Overthrown With Laughter \\
\hline Louise & $\mathrm{F}$ & 50 & "left of center" & Overthrown With Laughter \\
\hline Walter & M & $40 \mathrm{~s}$ & "three steps left of Stalin" & Overthrown With Laughter \\
\hline Suzie & $\mathrm{F}$ & 42 & liberal & Overthrown With Laughter \\
\hline Robert & M & 59 & "leftist" & Overthrown With Laughter \\
\hline Saul & M & - & conservative & Poor Get Poorer \\
\hline Brian & M & 66 & "way right of center" & Poor Get Poorer \\
\hline Liz & $\mathrm{F}$ & 68 & conservative & Poor Get Poorer \\
\hline Dora & $\mathrm{F}$ & 58 & libertarian & Poor Get Poorer \\
\hline Shawn & M & 34 & $\begin{array}{l}\text { "between conservative and } \\
\text { libertarian" }\end{array}$ & Poor Get Poorer \\
\hline Michael & M & $40 \mathrm{~s}$ & liberal & Overthrown With Laughter (blogger) \\
\hline Paul & M & $50 \mathrm{~s}$ & libertarian & Poor Get Poorer (blogger) \\
\hline
\end{tabular}


subjects of analysis, for two reasons. First, the focus here is on bloggers' and readers' experiences of blogging, not the digital artifacts produced. Second, analyzing the content of the blogs and comments would provide a deceptively incomplete picture, as such as analysis would not capture the interactions that happen via email and other media, nor would it include experiences of the act of reading. This is not to say that the blog content is unimportant, but rather that it is not the focal point of this study. The goal here, then, is not to examine the digital products of blogs and comments, but rather the social processes of blogging and commenting through participants subjective experiences thereof. Thus, reading the blogs and the comments enabled asking about recent posts, and it also provided a fuller context in which to interpret participants' responses.

Analysis of these interview data followed an iterative open-to-axial coding approach (J. Lofland \& L. Lofland 1995). After completing and transcribing interviews with each reader, transcripts were read and coded for emergent themes independently by each of two researchers. These researchers then met, discussed the themes each had found important, and developed axial codes that codified common themes identified by both researchers. These axial codes were divided between the two researchers, who wrote short, summary descriptions of what each code was and how it might pertain to the larger analysis of blogger-reading interactions. Codes pertained to general blog reading practices, such as choices of when to comment or how readers perceived others' comments, as well as political aspects, such as how readers constituted their own political ideology in relation to the blogs they read. In order to obtain more data about some of these analytic areas, we solicited a subset of our participants for a second interview, guided partially by follow-up questions from the first interview and partially by the coding process. Interviews questions with the bloggers were also guided by the emergent themes represented by codes. After conducting and transcribing all interviews, all transcripts were coded following the axial coding scheme using the TAMS Analyzer software. ${ }^{3}$ This final coding process was still iterative. Some axial codes, such as those pertaining to political ideology, ended up warranting further subdivision; reading blogs of differing ideologies, the explicit ideologies of bloggers compared to the implicit ideologies in most MSM, and the various ways in which readers defined their own political ideology each ended up warranting separate codes. The findings presented below are based on the most prominent codes, the themes from which they are derived, and how each of these themes explicates an aspect of the ways in which bloggers and readers both participate in creating a blog.

\subsection{Participants}

Responses received to the recruitment posts not only included emails to the researchers but also comments on the left-leaning blog, and they provided an early insight into the tenor of discussions among this group of blog readers. 
Comments included jokes, sarcastic scoffing at the "chance" for a \$100 Amazon gift card, and questions about the researchers' source of funding. In the interest of not completely dissuading all readers there from participating, one member of our research team replied to these comments with a mix of actual answers and attempts at dry humor. This exchange provided a useful introduction to the tone and personality of that blog's commentariat. While the right-leaning blog does not include a comment feature, participants did not express similar feelings of skepticism. When asked about the post recruiting readers, for example, one reader "thought, 'oh, that sounds interesting,", and another saw participation in the study as his "way of giving back to [the blogger]." Since all readers participating in this study became involved by responding to a post on a blog, it may be that they are more interactive than the average blog reader. However, since the focus of this paper is interactions between readers and bloggers, those readers who voluntarily respond are likely the best participants.

Ten readers were recruited, five from each of the two blogs. All names related to participants - of readers, bloggers, and blogs - are pseudonyms. Five readers (two female) were from "Overthrown with Laughter," a progressive blog written by Michael, a writer, with witty tongue-in-cheek political commentary, and described themselves as liberal, left-leaning, or moderately socialist. Overthrown with Laughter averages about 2.3 posts per day and about 15 comments per post, many of which are from regular commenters or Michael himself responding to other comments. Five readers (two female) were from "Poor Get Poorer," a libertarian blog written by Paul, an economist, focusing on both personal and macro economics, and identified as either conservative, right-leaning, or libertarian. Poor Get Poorer averages about 1.6 posts per day and does not have a comment section. However, Paul regularly appends to his posts opinions, reactions, or links emailed to him by readers. While we would have preferred that both blogs include a similar commenting feature, the difficulty mentioned above in finding a conservative blogger willing to participate, combined with the frequency with which Paul posts content from readers emails and Paul's own description of this practice as a highly-moderated comment section, led us to accept Poor Get Poorer as a field site. This disparity in commenting features makes difficult other comparisons between the two blogs, e.g., asserting that differences in ideology impacted differences in readers' experiences. However, while subject recruitment specifically targeted blogs on both sides of the political spectrum, the purpose was not to compare the two, but rather to help provide a more complete picture. Indeed, in terms of the blogger-reader interactions that are at the focus of this study, ideology did not seem a crucial differentiator. Readers ranged in age from 34 to 68 . Most were middle class to upper middle class, selfidentified as white or Caucasian, were relatively tech savvy, and had been reading blogs regularly for at least a few years. As noted above, while a small, relatively homogeneous group of participants may impact this paper's ability to make generalizable claims, it enables a much richer, deeper understanding of the 
subjective, lived experiences of interactions between readers and bloggers. Capturing those experiences is the goal of this study.

\section{Findings and Discussion}

The focus of this paper is to understand how the interactions between readers and bloggers affect the activity of blogging. The analysis presented here suggests a critical consideration of the analytic categories of reader and blogger. Specifically, casting an individual as a reader implies that $\mathrm{s} / \mathrm{he}$ does not have a major role in shaping a blog. However, the findings here indicate that readers do play a significant part in the creation of a blog. This section explores the various ways in which bloggers and readers together participate in the activity of blogging through a number of issues:

- Do readers feel that they are participating members of a community, and how does that participation shape the blogs they read?

- How do reading habits and choices, political ideology, and political participation relate to one another?

- What differences do readers perceive between mainstream media (MSM) and social media, and how do these differences mediate readers' impacts on blogs?

This paper argues that the process of blogging is distributed across the activities of various individuals and groups spread over time. In order to study and understand the phenomenon of blogging, we look not only at the various activities of which it is composed - reading, writing, commenting, emailing, etc.but also at the various interactions between these activities and those who engage in them. The following subsections detail examples of such activities as described by our participants. The subsequent section synthesizes these findings, arguing that each demonstrates a specific way in which blogs are collaboratively co-created.

\subsection{Community, Membership, Participation}

Questions of community from a reader's perspective revolve largely around membership and participation. Do I belong to this community? Do I participate and contribute? Are my contributions significant? Rather than consider the notion of community per se, this section instead looks at the particular components of how readers constitute a sense of community, and how various forms of participation in that community become means for bloggers and readers to collaboratively shape the blog through their interactions. There is also a resonance between the notion of "being a part" of a blog (cf. Baumer et al. 2008) and blog reading as a form of political participation, which is explored further in Section 4.2.1 below. Being a part of a blog, however, is distinct from feeling as if one is part of a community; the notion of "being a part" of a blog refers specifically to the reader feeling as if $\mathrm{s} / \mathrm{he}$ has a hand in shaping the blog, rather than community membership. 
While we begin this discussion with participants' invocations of community, the end of this subsection returns to problematize applying the notion of community to blogs.

Our readers considered participation as an important requirement for membership in a community. If Shawn is "attracted to read the blog then [he] participate[s]... it's like a community being formed." Nonetheless, the feeling of membership also heavily relies on the blog reader's definition of participation. Louise participates "by reading [the blog], by commenting occasionally, by linking to it, by forwarding the links to other people," and she "feel[s] like reading blogs and occasional commenting ... makes you part of a global community." Importantly, only occasional commenting is necessary for participation; what's more important is the general milieu of interaction around the blog. On the other hand, Liz does not feel that blogs are a source of community, because she "[doesn't] really participate in them," which, in her terms, means that she does not usually post comments. However, she regularly corresponds directly with bloggers she reads via email, which likely contributes to her feeling a close, personal connection with them: "when some of them go on holiday I just feel like, 'my God my friend's gone out of town, where is he?"' While they may not call it a sense of community, all readers described something similar to this sort of personal connection. "You never meet these people, ... but somehow they're still real and sometimes quite dear to you." This sentiment resonates with previous findings about participation and "being a part" of a blog (cf. Baumer et al. 2008), particularly the way that participation and community are constituted differently by different readers in different contexts. There is also a resonance between the personal connections seen here and the strong personal connections experienced by users of some DLs (Galegher et al. 1998), a resonance which is explored in more detail in Section 4.3.3 below.

From the blogger's perspective, readers are not only significant, they are indispensable. Paul regularly appears on television shows where, as he puts it, he is "on the same time as other guests and they deliberately pick guests who disagree with each other and they try to stimulate arguments and fights." After a number of such appearances, "readers started writing with these stories about these ludicrous, wrong market predictions [the other guest] made over the years," which Paul would then use as "ammunition" during subsequent appearances. From his perspective, "it's just great I've got all these thousands of pen pals who do that research for me." According to Paul, "one of the greatest advantages to having a blog is that I find I know stuff that I need to know without fail, instantly, 'cause there are enough people who know what I want to know and for some reason want me to know it and they tell me."

Here we can see that the reader has a significant capacity to affect not only the blog but also the blogger and her or his activities beyond the blog, a capacity which is discussed further below. However, despite the direct impacts they have, 
readers often do not feel particularly significant. According to Brian, "I don't know if I have any significance other than reading his stuff and I suppose I become a number that logs in to, you know, increase readership which allows him maybe to sell some ad space or something for his blog." Brian does recall emailing Paul about a "Joke of the Day" that never changed and lightheartedly stated, "I was getting a little tired of the joke....and as soon as I said that [Paul] changed his jokes a couple times," but he downplays the significance of this event. Similarly, when asked how important he was as a reader, Walter responded, "Meh, only important to me. I show up as a hit on their webalyzer." This perception can be seen as arising from the asymmetry (A. Voida et al. 2008) in blogging, that blogs are, both technologically and socially, focused on the blogger. This is not an argument that such asymmetry should be avoided or designed away, but rather a possible explanation for readers' perceptions of their own impact.

Another possible contributing factor is that, on their own, each individual contribution from a reader may not seem terribly significant. A single factual correction, link to relevant information, or a prompt to change the Joke of the Day may not, by itself, amount to much. However, while each reader sees her or his individual contributions as not being terribly significant, these "microcontributions" in the aggregate add up to be invaluable for bloggers.

Part of this feeling of insignificance may also stem from the invisibility of inaction. In physically collocated interaction, it can be readily apparent when individuals pointedly ignore and do not react to actions of others. However, such inaction towards unwanted behavior is not as readily apparent on blogs. For example, five of our participants became frustrated with unwanted comments, including "confrontational [comments where] each person wants the last word," comments that "degenerate into praising the blogger or people making their own jokes," "criticisms and ridiculous stuff and personal insults," or "TLDR [too long don't read]." In some cases, readers would completely avoid the comments section; "it just became something I didn't want to put in my head." In others, readers would only look at the comments section when seeking clarification about something in the post. Some of these reactions to undesirable behavior resemble those of lurkers who feel shunned or who feel that a particular group is rude and unapproachable (Preece et al. 2004). In DLs, such attitudes can drive away new members and potentially lead to the dissolution of the group due to lack of participation. Inaction in such contexts can be quite meaningful; a lack of response is a type of response, one of dislike, disapproval, or disinterest. In the context of blogs, however, such inaction is either less visible or ambiguous as to whether other readers are not reading the comments, are reading but not replying to certain commenters, or are not reading the blog at all. Since reading alone without explicit interaction is an acceptable central form of participation blogging, it becomes difficult to spot explicit inaction and, moreover, to determine what that inaction means to the inactor. Not only does this potentially 
interfere with the collaborative co-creation process between bloggers and readers, but the invisibility of such inaction may also contribute in part to both the slow norm formation noted in blogging (Lenhart 2005) and readers' feelings of insignificance.

The disconnect between readers' self-perception of significance and the blogger's perspective of readers' significance points to problems in defining the term "community." Framing blogs as a site of community necessitates questions of membership, which, even when fluidly defined, is often perceived and constituted differently by different individuals; what counts as membership for one person might not for another. For example, Liz felt that membership in a community required some type of active and regular contribution, such as commenting. Thus, even though she emails bloggers frequently and feels close personal connections with them, she does not feel a sense of community. Furthermore, this view unnecessarily homogenizes the medium; readers' interactions around blogs vary widely and may or may not fit into traditional notions of community (Baumer et al. 2008). Rather than viewing blogs in terms of community, it may be more informative, as we have done here, to examine how readers participate in - are a part of - the blogs they read, the various ways in which participation is constituted, and the role that reader participation plays in shaping a blog.

\subsection{Political Ideology, Reading Habits, and Political Participation}

Previous studies examining the selection of online news sources have indicated that individuals demonstrate homophily, generally seeking information that resembles or resonates with their political opinions (Farrell et al. 2008), and, while they do not often expressly seek out conflicting views, they do not avoid such conflicts, either (Garrett 2005). In agreement with this previous finding, all of our participants predominantly read blogs with whose political ideology the agreed, and they do not avoid reading blogs whose ideology conflicts with theirs. However, in contrast to the non-avoidance behavior of Garrett's subjects, six of our ten readers regularly read, explicitly seek out, or have sought out in the past, blogs of different political ideologies than their own. This behavior is often justified as a chance "to hear what other people think" or "just to see what they're up to" on "the other side." Generally, readers do not "religiously follow" such blogs, but rather, as Dora puts it, check in "at least once every couple weeks ... to see what the people there are saying." This practice may also contradict Farrell et al.'s (2008) findings about homophily. Farrell et al. qualify their result slightly, noting that, because their data about which blogs subjects read comes from a text box with a 64-character limit, it is possible that did not have room to list all the blogs they read regularly. Thus, it may be that more individuals read across party and ideological divides than their data indicate. However, they suggest that homophily is the more likely conclusion, as not many of their data points seemed 
to be affected by the character limit. We suspect, though, that Farrell et al.'s subjects listed only those blogs they read regularly, rather than every blog they read at all.

Those of our participants who did read across ideological lines did not do so as part of their regular reading routine, but rather simply to check in on the "other side" occasionally. This practice was not universal, though. Liz "stopped reading [some blogs] because they just started being too anti-Bush for [her]." Walter used to read conservative and libertarian blogs but claims, "I could feel myself getting dumber." When cross-ideological reading occurred, participants it described more as an act of voyeurism or perhaps espionage than they did with their more routine blog reading; readers generally do not feel a part of these blogs. Indeed, Gilbert et al.'s (2009) results suggest that, if such reading across ideological lines does take place on a broad scale, those readers are not leaving comments, at least not comments that expressly disagree with the blog post. On the political blogs in their sample, comments agreeing with a post outnumbered those that disagreed 9 to 1 (though only $60 \%$ expressed explicitly agreement or disagreement). However, the political blogs in their sample were all major, A-list blogs, including Huffington Post, Daily Kos, and Think Progress. Gilbert et al. argue that their sample is more likely to evidence cases of disagreement, since "blogs that give voice to many and are read by many" (Gilbert et al. 2009, p.3) are more likely to act as public spheres (cf. Habermas 1991). However, the highly polarized major political blogs do not necessarily represent the dispositions of most political blog readers.

While some participants self-identified specifically as liberal or conservative, making a clear distinction into one side or another was not easy for many of our participants. "Of the bloggers" on multi-authored blogs, "there isn't anybody on there that [Eli] agree[s] with, so it's hard to develop a relationship with that blogger." Again, we can see that a relationship with the blogger being of pivotal importance. Moreover, this is an example of a reader unable to place himself within the commonly used parameterization of politics into liberal and conservative. Furthermore, readers even experienced situations where blogs with views similar to theirs would post something with which the readers disagreed.

This suggests that traditional ideological divisions along the lines of conservative versus liberal are not only being questioned by blog readers but found to be inadequate. Participants identified themselves or the blogs they read as "right of center," "sensible conservative," a "conservative ... Kennedy Democrat," "somewhere between conservative and libertarian," or "what might be considered slightly socialist in Europe [but] here would be considered three steps left of Stalin." When trying to describe her own views, Louise says, "I don't know, where do you draw the line? I guess it's a circle so eventually things kind of meld into one another." Some users felt marginalized enough by their particular political views that they avoided strongly partisan sites like Daily Kos, 
even when they agreed with them. Farrell et al. (2008) argue that the combination of such highly partisan blogs with readers' tendency for homophily could reduce the degree of exposure to alternate viewpoints and subsequent deliberation. However, the findings from our participants suggest that some readers' aversion to such strong partisanship might suggest more room, and possibly more desire, for deliberation than evidenced by Farrell, Lawrence, and Sides' data. This may also be due to the possibility, noted above, that their participants entered only the blogs they read regularly, rather than including every blog they ever read. While the currently available categories for framing political debate are not necessarily problematic per se, as Louise puts it, "they obscure more than anything else." These traditional categories may be somewhat revealing, but they do not tell the whole story. While immediate alternatives are somewhat unclear, the discussion section below considers some possible options.

Coincidentally, during the time when this study was being conducted, the social networking site Facebook changed the way that users list their political views in their profile. Where users previously chose from a spectrum ranging from very conservative to very liberal with an option for libertarian, they are now given a text box that automatically suggests political parties from around the world but that also allows any free text to be entered (Losse 2008). That readers find a single-axis ideological spectrum limiting is hardly surprising. However, it might cause us to question previous findings about selectional exposure (Garrett 2005) and homophily in blog reading (Farrell et al. 2008), partly because determining similarity of political views is difficult when individuals do not strongly identify with a single, established political ideology; partly because a reader, even if s/he agrees with a blogger's opinion, may not appreciate a strongly partisan, one-sided presentation of that opinion; and partly because, for our participants, political viewpoint, while certainly a factor, is not the primary determiner of which blogs to read.

Ultimately, why a reader chooses to read specific blogs is influenced not only by ideology but also by writing style. Suzie may be "drawn to [a blog] because of the political point of view but [she] won't keep reading if its not good writing." Similarly, Louise "[doesn't] have a lot of tolerance to read somebody's work if they don't know how to write." As in a previous study of blog readers (Baumer et al. 2008), humor and entertainment, including "witty commentary and critiques," were important motivators. "Over the top with a sense of humor gets read. Over the top without a sense of humor does not." However, not all blogs needed to be humorous. "It's like owning a stock portfolio," says Brian, "you like to have some of different things." This is not to say that political ideology is unimportant. Rather the point here is twofold: that a blog's political ideology is not the only important factor to a reader, and that the ways in which ideology is important are complex, nuanced, and interrelated to readers' interactions with a blogger. 


\subsubsection{Reading as Participation}

In their survey data from blog readers, Farrell et al. (2008) found that readers of political blogs were significantly more likely to participate in terms of voting, donating to a candidate, or persuading another person to vote for a particular candidate. Among the participants in our study, all politically participate by voting, and six are politically active in additional ways, several of them donating money or writing to their representatives. Brian serves as a poll worker, and, during the 2008 Democratic primaries, Suzie went so far as to "hit every house in [her] precinct with a little card that tells them what precinct they're in, where their caucus is, and how the Democratic caucus works in the state." Since our participants care enough about politics to read political blogs regularly, and given Farrell, Lawrence, and Sides' results, it is not entirely surprising to find that many of them are involved politically.

However, our participants also view their reading itself as a form of political participation and engagement (cf. Garrett 2006; McKenna and Pole 2004). According to Eli, a "blog affects citizens and encourages citizens to be involved and stay up on the issues;" it "encourages people to get involved." Dora is "not the kind of person that goes to rallies, or even has ever worked for a candidate," but "[she] pay[s] a lot of attention," partly through reading blogs. Similarly, Shawn is "always eager to engage in a conversation but [he's] not that likely to go and sell t-shirts ... or stuff like that." Based on survey data, McKenna and Pole (2004) argue that bloggers perceive blogging as a form of political participation. Drawing on Kim et al.'s (1999) notions of campaigning and complaining, Walker (2006) found instances in comments on political blogs of campaigningencouraging others to become involved in the political system-but not of complaining - voicing of opinion outside the political system. Walker's finding also resonates with that of Gilbert et al. (2009) in terms of the low levels of disagreement, as well as those of Farrell et al. (2008), in that homophilic readers are less likely to disagree with what they read.

However, among readers of relatively similar ideological positions, there is still opportunity for deliberation, even if the result is not radical changes in viewpoint. "No one has left their family or job because of something I said," says Walter, "but there's a constant dialectic where we all change our positions slightly based on things we discuss." This "constant dialectic" is the sort of deliberation that Farrell et al. (2008) argue can be fostered among readers of blogs with varying political ideologies. It may be that our participants are among that small percentage who do read across ideological lines, it may be that there are fewer strictly homophilic readers than previously suggested, or it may be that even reading political blogs where one agrees with the blogger may encourage political deliberation and debate. The dynamic interactions of blogging help to enable this constant debating and shifting of positions, as well as to provide a sense of blog reading as a form of political participation. Part of their feeling of political participation comes from "being a part" of the blog. By participating in the 
collaborative process of co-creating the blog, readers are also participating in the process of political discourse, debate, and deliberation in and around the blog. These co-creative processes act as important differentiators between social media, such as blogs, and more traditional mainstream media.

\subsection{Mainstream Media, Social Media}

Blogs and other social media, which often exhibit a personal style or flare, are sometimes seen as an alternative to traditional or mainstream media (MSM). MSM are those media produced by major news corporations, agencies, or conglomerates, such as CNN, Fox News, or National Public Radio (NPR). To be sure, there is not a clear distinction between MSM and blogs. Call-in radio, letters to the editor, and media personalities such as Paul Krugman or Glenn Beck make the boundary quite fuzzy, as shown in participants' experiences described below.

We rarely asked participants explicitly to compare blogs with MSM. Rather, participants volunteered these comparisons in response to other questions (e.g., "Why do you read blogs?" "Do you use blogs as a source of news or information?"), with eight participants citing differences from MSM as a motivation for reading blogs. This framing can make it seem as if blogs define themselves, and derive their significance, via differentiation from traditional media. While this may to some extent be the case, seeing these two forms of media in opposition to each other, or trying to make clear distinctions between MSM and blogs, not only hides the heterogeneity of blogs, but it also ignores some important ways in which blogs and MSM can be quite similar.

\subsubsection{Differences}

Seven of our participants used blogs rather than MSM as their primary source of news and information; Eli says, "I don't know what I'd do if they didn't exist." Furthermore, blogs can sometimes either break stories before other news sources or cover information not available elsewhere. Dora remembers a situation "with Drudge [of Drudge Report] back with ... Monica Lewinsky, when a couple of the magazines, the Times, they had information on it and they were going to sit on it. And Drudge didn't." Liz cites "the whole Canadian thing with Barack Obama and him saying, you know, that he went to the Canadians and said, 'don't pay attention to my anti-NAFTA because I'm not going to go there' ... That all started on the blogs."

This sort of quick response is a key component political blogs' impact. Farrell and Drezner (2008) argue that blogs gain a great deal of their influence because members of the MSM consult them for information, opinion, and commentary. Some of this immediacy also comes from reader interactions, as in the example described above where Paul's readers regularly email him with information and counter arguments as "ammunition" for his television appearances. A combination of blogs' low cost of publication, personal connections between bloggers and 
MSM, some bloggers' narrow but highly developed expertise, and blogs' capacity for nearly real time interaction in combination lead journalists to read blogs, which results in blogs having a great deal of influence on the media, and, thus, on the larger population of those who don't read blogs. Drezner and Farrell (2004) and Wallsten (2005) present studies of how A-list and "ordinary" political blogs, respectively, have varying and occasionally high degrees of influence on MSM. As described above, while Wallsten points out certain correspondences between the issues covered in MSM and blogs, he also indicates issues, such as immigration, affirmative action, terrorism, No Child Left Behind, and welfare, where the amount of coverage in political blogs different greatly from the amount of coverage in MSM.

Blogs also have many features attractive to readers as a news source not found with MSM. For some, the blogger's style, including "solid information and insightful commentary," are major motivators. As Louise puts it, "I really thirst for [bloggers'] commentary and their insights because it's just a refreshing change from the crap that you read in the media." Humor and entertainment value are an important part of that style: "[one blogger] knows how to use satire and sarcasm and makes things humorous, it's quite witty." Also in opposition to many MSM outlets, which generally claim to provide "fair and balanced" coverage, bloggers tend to openly and clearly state their political views and biases, which readers appreciate. One participant had "long been aware of biases in ... the New York Times, but there weren't really any alternatives." For this participant, reading blogs has provided an "eye opening" alternative.

\subsubsection{Similarities}

However, while they do provide an alternative, blogs are not always completely different from MSM. For example, Eli expressly avoids large blogs, "like, you know, Daily Kos is like the most famous thing," but "there's so much crap on there..., there's so much junk that I don't get anything out of it." Also, some large blogs have extreme, highly partisan ideologies, such as, again, "blogs like DailyKos [which] are considered super left," or "on 'America Blog'..., [whose] whole shtick is, you know, whatever Republicans do is bad and whatever Democrats do, by definition, is good." As noted above, such single-minded partisanship deters some readers. While there is a difference between an explicit bias and an unstated bias, Louise "disagrees with that whole mindset" of a single, permeating ideological viewpoint. Here, large blogs begin to take on qualities of MSM, demonstrating the heterogeneity of blogs and the interactions surrounding them. The distinction between MSM and blogs is further blurred when large news sources, such as the New York Times, have blogs on their website. To Suzie, such forays of traditional media into blogging seem "just kind of silly," "it just seems like they're trying to glom on without really understanding the medium," similar to "people that put up blogs for the sake of letting people comment except they cut out everything but the positive comments,... it's not very substantive." 
According to Walter, such blogs "are crap trying to get legitimacy in a new medium. $[\mathrm{MSM}]$ are dismayed at their lack of influence and control so they try blogging." The Wall Street Journal or New York Times can call a portion of their website a blog and include all the technical aspects common to blogs-frequent posts, comments, trackback links - but such efforts lack a key component of blogs: their interactional aspects (Baumer et al. 2008).

\subsubsection{Interaction and Connection}

A key part of such interactional aspects, and an important differentiation from MSM, is a personal connection with the blogger. Many readers interact with bloggers, either through leaving comments, or, more often with our participants, via emailing the blogger. Nardi et al. (2004a) also found that many bloggers would interact with readers through email. As noted above, of the two blogs from which we recruited participants, Overthrown with Laughter allowed for comments while Poor Get Poorer did not. However, the inability to comment did not impede interaction between readers of Poor Get Poorer and the blogger Paul. Of our 10 readers, 8 have emailed bloggers with comments about posts or specific questions. Even when comments are available, some readers are like Liz: "I tend to email the blogger directly as opposed to ... where I can be seen commenting." Sometimes, the blogger will reply simply with "hmm, interesting," or "thanks, interesting thought." Other times, replies are more verbose. For example, when Brian asked "[Paul] questions about calculating the various the [sic] type of numbers that go into how to measure the possibility of recession," he replied with "about a four to five sentence expression" describing "average wages and average productivity..., and how they relate to each other." In some cases, readers will email corrections or supplemental information to bloggers, which can result in the blogger making a change on the blog, often giving credit to the reader. Sometimes, they will "have a dialogue back and forth" about topics of mutual interest. Such interactions can lead to close, personal connections between bloggers and readers. For example, Louise used to read a blog called Memos on the Margin. "I had a couple of e-mail exchanges with the blogger, and I really ... felt quite bereft when one day I checked his site and there was this notice here saying that he had died of a heart attack." This close connection resulting in a feeling of bereavement occurred purely as a result of her reading the blog and their interactions via email. Galegher et al. (1998) found similar feelings of close personal connections among members of Usenet support groups for arthritis, attention-deficit disorder, and depression. However, Galegher et al. did not find evidence of such feelings among members of hobby groups based around cooking, pet dogs, and quilting. Topically, the political blogs in the present study are more similar to the hobby-oriented Usenet groups; our participants did not describe the sharing of personal life details, such as struggles with depression, as a central component to their blog reading. Nonetheless, readers still felt a close affinity for many of the bloggers they read. 
Interactions can lead to important relationships from the blogger's perspective as well. In the more than 6 years that Paul has been blogging, he has "created extremely valuable relationships ... often people [he's] never met face-to-face and probably never will." Some end up being business partners, some end up as contacts or sources of information, and some end up as friends. Similarly, Michael has "made a ton of professional contacts - editors will know me before I pitch them stories" - in addition to personal friendships. "If blogs did not come with comments I would have stopped doing it a long time ago." This sense of community arising from a connection with his readers is a major motivation for his continued blogging.

However, a connection between blogger and reader need not be made explicit through direct interaction with the blogger. Some readers who email bloggers only do it "every once in a while." Dora said, "I think maybe once I wrote to Paul, ... [but] I don't necessarily feel that I have anything very interesting to contribute more than other people." In this case, the blogger's style - descriptors for which included "breezy," "opinionated," "humorous," "pithy," "witty," "sharp," "satirical," and "incisive" - can give the reader a sense of who the blogger is as an individual without the blogger necessarily sharing details of her or his personal life. Furthermore, bloggers often incorporate or discuss aspects of their offline life in the blog. For example, Paul is a known financial analyst, appearing regularly on TV shows, and he includes information about his professional life on his blog. Interestingly, most participants referred to Paul's blog as "Paul's blog" rather than Poor Get Poorer. On the other hand, Michael has almost no mention of his offline life on the blog, aside from advertisements for his recently published book, and many of his readers referred to his blog by name, either "Overthrown with Laughter" or simply "OWL," rather than as Michael's blog. This difference has implications with respect to whether readers see the blog as the digital instantiation of the blogger (Reed 2005) or as an object that belongs to the blogger, as well as whether they connect primarily with the blog or with the blogger. These connections are another important piece of understanding how blogger-reader interactions shape the blogging experience, both for readers and for bloggers.

\section{Discussion and Synthesis}

The core argument presented here is that the creation of a blog is a collaboration activity in which both bloggers and readers engage. The primary contribution of this paper, then, is developing a grounded understanding of the specifics of how these collaborative co-creation processes are conducted. This section presents further, specific examples detailing the interactions between bloggers and readers, highlighting the ways in which readers contribute. The section concludes with an argument for this finding's significance, as well as its various implications: for understanding how readers contribute to shaping a blog, for the development of 
technologies to support both bloggers and readers, and for theorizing the social processes of social media.

\subsection{Bloggers and Readers Blogging Together}

A blog is a discussion, a conversation, an exchange between bloggers and readers (Baumer et al. 2008). An important aspect of a conversation is that it is shaped by all participants. In many cases, a blog is followed by a consistent group of readers. Through commenting, readers recognize and remember one another. For Walter, an important aspect of Michael's blog "is that there aren't 100s or 1000s of commenters. You can get a sense of personality from the commenters because most comments are self-expository." Louise notes that "there seems to be a community of readers on certain blogs like Overthrown with Laughter ... that post quite good comments. Sometimes they're almost as informative as the post itself." Not only do these comments contribute content to the blog, but they also help set the tone. Brian describes how commenters "poking fun at each other" makes reading "like being in a community-type-thing." Through sending emails back and forth with the blogger, Shawn recognizes that he "help[s] shape the blog." Even for Liz, who does not often comment, "interaction is important in the sense that I want people who are there doing something." In other cases, commenters "just detract, [there are] total nutcases and people who just go out of their way to be deliberately provocative and offensive." On OWL, "[t]here appear to be a mix of MIT, Yale, Harvard, Princeton semi-intellectuals with a good sense of humor," creating what Walter calls a sort of "collegial" atmosphere. This same atmosphere causes Eli to be hesitant about commenting there: "a lot of the [commenters] are very funny, so I'm afraid I'm going to come off, on the one hand, as too serious and I'm going to spoil the fun, and on the other hand I'm going to come off not knowing enough." His hesitance resembles some lurkers' fears about rejection or saying something others will think is stupid (Preece et al. 2004). Also like lurkers, whose posting patterns vary between discussion lists, Eli will post comments to other blogs he reads. However, he attributes this difference to the tone and style set by the blogger, not the other readers. One blogger, profcolfax, is "not entirely up-to-date the way that other bloggers are," says Eli, "I don't feel as dumb commenting there." However, while Michael may in part set the tone for OWL, it is also in part the other commenters whose humor causes Eli's hesitance. The sarcastic comments in response to our post recruiting participants provides a prime example of this caustic humor. In OWL, we can see that bloggers and readers together set the tone of a blog and associated comments.

Readers also perceive that comments must be made in a timely fashion. Eli recounts an example where he would have commented but "didn't see [the post] until ... 3 or 4 weeks after the fact..., so I didn't see it in time." "OWL locks comments after 7 days," notes Walter, "so getting interaction with other commenters is most likely while the post is fresh." However, like oral 
communication (cf. Ong 1980), "blogposts and comments are transient," not something to which consistent, sustained attention should be paid. While a primary motivation may be "interaction with other commenters," that interaction itself becomes part of the process of shaping the blog.

Many readers of blogs are often bloggers themselves (Baumer et al. 2008). Of our participants, only Suzie regularly maintains her own blog. Walter has a blog but does not post regularly, Louise used to have a blog but "it's kind of on hold at the moment," and Eli started his own blog as a result of participating in this study, citing the fact that he wanted a place to vent his own opinions without needing to comment on someone else's blog. For Suzie, reading other blogs affects how she blogs. For example, "the one thing that will make a blog reader mad ... if somebody changes their format, some people will like it but the majority will hate it." Also, when quoting another item, "I always include links to a story wherever I got the quote," because when reading, a link to a source "stops me from having to search around." While these are somewhat pragmatic details of how a blog is organized, they demonstrate that the activity of reading is closely connected to, and influences, the activity of writing.

Similarly, one might assume that bloggers also read other blogs. With Paul, however, this is not the case. "I used to be just obsessive. When blogging first started..., it was so cool and there weren't that many of them, but I just kind of got tired of it." Part of this may have to do with his definition of what constitutes a blog: "the essential definition is the idea of continuous real time refreshing and new content ... that you have basically a continuous rather than an interval publishing schedule," regardless of whether it is an individual or a group, whether it is independent or part of a news source such as the New York Times, whether it has a personal style, or whether it undergoes a rigorous editing process. With this definition, the major motivation becomes reading for those regular updates, for the current information. However, Paul no longer needs to read blogs or any news source to stay current, and in fact gets a much higher ratio of information in which he is genuinely interested without actively or regularly reading other news sources. This up-to-date information comes from his readers. Paul receives "a constant stream [of email] all day, everyday, dozens of them," from his readers, and he classifies those emails into three groups. Emails in the first group, he says, "point me to things they just think I might like to read," to which he sometimes posts links from his blog. The second group "actually provides content," information submitted anonymously, sometimes from Washington insiders, to whom Paul only refers using pseudonyms. The third group of readers will submit replies to or thoughts about posts he has made and, if the reply is particularly noteworthy, he adds it as an update to the original post, "which is really just a sort of a manual version of a comment section." One of the reasons that Paul communicates with his readers so much via email may be that he does not have a comment feature on his blog. Not only do those readers email him who would normally email a blogger, but he likely receives emails from readers who would 
normally comment, too. This is not to say that everything he receives from readers is valuable and insightful. "A lot of people ... assume that I am a conservative of the Rush Limbaugh type and ... turn me onto a lot of this real classic right-wing stuff that just doesn't interest me at all." However, using the social filtering of his readers' references still results in a reduction of the amount of junk through which he would sort: "you read the New York Times every day you get a thousand times more..., it's not filtered at all." Paul certainly sees this degree of availability to his readers as an asset, which may call into question previous findings that bloggers often desire to limit the level of interactivity between themselves and their readers (boyd 2006; Gumbrecht 2004; Nardi, Schiano \& Gumbrecht 2004).

Not only do these examples demonstrate that readers make integral contributions to the blogs they read, but the experiences that Paul, Michael, and their readers describe here show just how clearly collaborative this process of cocreation is. This is not to argue that bloggers and readers contribute in equal and identical fashion to creating a blog. The two certainly play different roles at different times, roles that are delineated both by social norms and by technical constraints, but each of these roles is important and significant in its own way. Readers provide the blogger with general feedback, give the blogger suggestions about relevant material, help correct mistakes the blogger might make, and even at times write content that becomes integrated with posts on the blog. Furthermore, readers provide a sort of looking-glass self (Cooley 1902) for the blogger. Links from readers directing Paul to "Rush Limbaugh type ... classic right-wing stuff," while they might not be of interest to the blogger, help give him an impression of how readers perceive him. Similarly, the humorous, witty, sarcastic tone adopted by the commenters on OWL indicate to Michael what sort of discourse his readers see as appropriate and, moreover, the ways in which readers perceive the identity he presents through the blog. Furthermore, in order to understand the blogger's self-presentation, one must look not only at interactions through the blog but also those outside the blog, such as Paul's detailed response to Brian's question about figures for calculating the likelihood of a recession. Not only do readers share in co-creating the blog, their perceptions, and the actions they take based on those perceptions, are also in part responsible for co-creating the identity of the blogger.

\subsection{Implications}

The data and findings presented here demonstrate that the bloggers and readers in our study do in fact engage in a collaborative co-creation process. This result has significant implications for studies of blogging and of many other social media. Any study of blogs, bloggers, or blogging that does not take into account interaction with readers, whether that interaction occurs through the blog or outside of the blog, necessarily limits itself in terms of understanding the social 
practices of social media. This section describes in more detail the implications of the findings presented in this paper. Specifically, it describes implications in terms of understanding the ways in which readers shape a blog, of developing technologies for bloggers and readers, and of advancing theoretical accounts of social media.

\subsubsection{How Readers Shape a Blog}

The most productive way to understand how bloggers and readers interact is to examine the processes by which they both contribute to the creation of a blog. Our data suggest three specific ways in which readers shape the blogs the read: by contributing content, by setting the tone, and by constructing the blogger's identity. This is not meant to be an exhaustive list of the ways in which readers can impact a blog. Rather, it provides specific, concrete means by which future blogging work can take readers into account, i.e., by asking the questions described here.

First, how do readers contribute content to the blog? In our study, reader contributions came primarily via comments and emails to the blogger, but other sociotechnical possibilities certainly exist. Although not present in this study, other work has noted that readers sometimes interact with bloggers face to face, providing feedback and suggestions (Nardi, Schiano \& Gumbrecht 2004). A blog could include a contribution form, enabling readers to suggest specific news items or general concepts about which they would like to see posts. Such a contribution mechanism could accept a variety of media, including pictures, video, audio, or RSS feeds. Many bloggers include widgets that present their Flickr feeds, and some have started including their Twitter feeds, on their blog. However, such tools could also be adapted to provide readers a means of contributing as well. The important part here is not necessarily to understand the artifacts themselves being contributed, but rather the sociotechnical processes by which those contributions are made.

Second, how are readers involved in setting the tone, both for other readers and for the blogger? Readers establish whether or not a given blog is one on which readers comment regularly, the types of comments that are appropriate, and whether or not readers interact with one another. In our study, readers set the tone mostly through comments. For example, Walter described how OWL's commentariat created a "collegial" atmosphere. This feedback also influences the blogger. Certain types of posts get certain types of responses; Michael's dry wit may be a hit with his readers or it may fall flat. This feedback need not come through comments, though. The emails that Paul receives about extreme, right-wing political material provide him with a sense of how readers perceive his interests, which subsequently influences the way that he frames future posts. In large part, this question of tone is a question of norm formation; in what ways do readers contribute to shaping the social norms for a given blog? 
Third, how do readers participate in constructing the identity of the blogger? This question is related to how readers set the tone, but has its own distinct and important implications. To some extent, the blogger does much of the work of presenting her or his own identity. Previous work explored the relationships between a blogger's identity presentation and readers' perception of a blogger (Baumer et al. 2008). However, readers do not just perceive a blogger's identity, but contribute to shaping that identity, as well. This shaping goes beyond the content and tone of the comments. By asking certain questions, such as how the likelihood of recession is calculated, or by making certain remarks, such as sarcastic responses to jokes, readers construct the blogger as the type of person of whom those questions are asked or to whom those kinds of remarks are made (and, reflexively, they construct themselves as the type of reader who asks such questions or makes such remarks). Even when those questions and remarks come via email rather than in comments on the blog, this looking-glass self continuously reshapes the bloggers perception, and in turn presentation, of her or his own identity.

\subsubsection{Technologies of Blogging and Technologies of Reading}

A large amount of work has been done on developing tools for bloggersBlogger (http://blogger.com), Word Press (http://wordpress.org), Movable Type (http://movabletype.org), and other publishing platforms and software are common examples. However, relatively little attention has been paid to the development of tools for readers of blogs. Some work has been done on tools to visualize collections of RSS feeds (Dennis and Jarrett 2005) and on methods for presenting different aspects from collections of RSS feeds (Hong et al. 2010). There remains, however, much work to be done on tools designed for blog reading. This work should focus on exploring how such tools can enable and support the co-creative processes described here; the preceding subsection presented one such concept, an explicit contribution mechanism for readers. Such work should also explore how reading tools might inadvertently prohibit or make difficult these collaborative co-creation processes.

Our findings about political ideology also pertain to technological design. Participants in our study sometimes read blogs of differing ideologies than their own, but they did not read those blogs in the same way; those were not blogs of which readers were a part. On the one hand, there might be a design space for tools that would promote exposure to different opinions. On the other hand, Farrell et al. (2008) argue that such broad and varied exposure can lead to decreased participation. The exact impact of such a tool on political deliberation is a question that should be addressed with empirical investigation. Another important aspect of readers' perceptions of ideology gives a slightly clearer inspiration for design. Most readers described their political views in ways that did not fit into traditional ideological categories. However, analytic tools incorporating blogs (Gamon et al. 2008; Baumer et al. 2010) have used very 
traditional distinctions, such as conservative vs. liberal and republican vs. democratic. As described above, these categories are not without their uses. However, an alternative to such externally-imposed categorization of blogs' political ideology might be a bottom-up, tagging or folksonomy-based approach. We described above how, during the time this study was being conducted, Facebook changed the political affiliation item in their users profiles, from a prescriptive drop down menu with limited options, to a free-text box for listing party affiliation (Losse 2008). Similarly, rather than classifying blogs along a traditional political spectrum, blog aggregation sites or analytic tools could allow readers to tag the blogs they read with more descriptive political ideologies, allowing for a blog to be a "conservative Kennedy democrat" or "three steps left of Stalin."

\subsubsection{Participation, Communities, Practice-Social Media Theory}

Previous research has at times likened online discussions to communities of practice (CoP) (Wenger 1998), where lurking can be seen as a form of legitimate peripheral participation (LPP) (Lave and Wenger 1991). Some general tenets from this tradition provided useful guidance here, in that they direct us to examine the ways in which social interaction serve to scaffold learning and understand, and the ways in which action is made meaningful via its situation in a social group. Indeed, the central argument of this paper is that, in order to understand the phenomenon of blogging, one should attend to the social processes involved in the creation of the blog. However, some analytic prerequisites do not apply in this case, drawing into question the notion that blog reading can or should be seen as a form of LPP in a CoP.

Specifically, a $\mathrm{CoP}$ is defined by three main aspects: the domain, the community, and the practice. In the current study, there is clearly a shared domain of political discourse among all participants. However, the other two aspects of a CoP are not as readily apparent.

First, as argued above, seeing the readers of a blog as a community can be problematic for a number of reasons. Readers of a blog do not hold explicit membership in a professional organization, such as cutters of meat (Lave and Wenger 1991) or insurance claims processors (Wenger 1998). LPP has been applied to lurking in DLs (e.g., Lee et al. 2006), but these lists have explicit membership. Even though not all members participate in the same way, one is either a member of the list or one is not. As described above, though, blogs do not have such a clear, crisp delineation of who is and who is not a reader. Rather, readership is defined in a much more fluid, discursive manner. For example, Eli does not read the blog OWL highly infrequently, but he still reads it regularly; that is, he does not check back every day, but he makes certain to read it every week or two. Does reading a blog only once a week mean that one is less or more of a reader than others? The point here is that readership of a blog is much fuzzier than membership in a professional or social group, and that while membership is 
generally explicated via relatively uniform standards, readership is continually constituted in a variety of ways.

Second, a CoP requires a practice in which members of the community engage, at first via LPP, and, eventually, via full participation. If blog reading were a CoP, we should see such a progression, from reading a blog, to emailing a blogger, to commenting on a blog, to creating and writing one's own blog. However, we do not see such a progression in our data. Unlike communities of Yucatan midwives or Alcoholics Anonymous members (cf. Lave and Wenger 1991), the purpose of a blog is not to help all its readers become bloggers in their own right. Rather, what we see is that bloggers and readers play various but equally important roles in the collaborative co-creation of a blog. If we see blogging as full participation and reading as LPP, this perspective fails to highlight the varied ways in which readers and bloggers both fully participate in creating a blog.

An alternative might be to argue that blog readers constitute their own CoP, distinct from bloggers. Readers would begin by only reading, then start emailing a blogger, and eventually comment regularly on the blog. Our data, however, suggest this may not be the most analytically illuminating approach. Seeing blog reading as the practice that defines a community of readers implies that a reader should work toward full participation in the co-creation of the blog. However, several of our participants were neither engaged in emailing and commenting, nor were they inclined to do so. Such reading alone, without other forms of interaction, should not be seen as LPP, but rather as another means of full participation in a different role. Furthermore, such an analysis would focus too exclusively on readers, thereby drawing attention away from the reader-blogger interactions that the findings presented here have shown are crucially important. Thus, we argue, LPP and CoP are not the most useful analytic notions to apply in understanding interactions between bloggers and readers.

A more recent approach to understanding various forms of participation in online media is the reader-to-leader framework (Preece and Shneiderman 2009). In this framework, users belong to one of four categories - reader, contributor, collaborator, leader - each with an increasing level of activity and investment in the group at hand. For example, in Flickr, a user may start off by viewing photos, then may contribute her or his own photos, then may work with other users to create sets of photos, then may organize groups of photographers around a common interest and work to enculturate newcomers. Preece and Shneiderman also note that an individual may move through these categories in a non-linear fashion, but such progressions are less common. This framework is somewhat useful here, in that it provides a way of seeing different blog-related activities, such as reading, commenting, or posting, as representing different levels or degrees of participation in the blog.

However, when thinking about blogger-reader interactions, the roles defined in Preece and Shneiderman's framework do not map clearly onto the findings in this study. Their "reader" role maps to the blog reader who does not comment or 
otherwise interact, and their "contributor" role might map to the blog reader who comments and/or emails the blogger. The "collaborator" is much fuzzier; perhaps this role would fit a highly active reader who contributes content to a blog but does not own a blog, but we did not observe such practices. The "leader" role is similarly problematic, since, as noted above, most bloggers are not invested in the process of making their readers into bloggers, nor do they frequently organize their readers as part of a larger enterprise. Thus, the roles do not capture well the blogging activities we observed.

Instead of a role-based approach, we have argued here for an activity-based approach. As described above, rather than seeing a given individual as a blogger or as reader, we look instead to how any given activity contributes to the creation of a blog, regardless of who engages in the activity. In this way, the reader-toleader framework can be helpful, not in terms of placing individuals into one role or another, but rather in terms of enumerating and categorizing different types of activities, all of which can serve as forms of participation.

While an alternative theorization of social media might be valuable, such an enterprise is beyond the scope of this paper. Instead, we suggest that further work can be informed by the findings presented here, possibly synthesizing them with previous work to suggest novel social media theories. For example, Wenger (1998) describes three modes of belonging in a CoP: engagement, which involves negotiating what constitutes meaning in the community; imagination, which involves envisioning the world in a certain way as framed by one's own experience; and alignment, which involves coordination among community members to make a contribution on some larger scale. In terms of understanding blogger-reader interactions, we suggest that the three forms of reader participation described above - contribution content, setting the tone, and constructing the blogger's identity - could inform theorizing about these interactions. Such a perspective would seek to understand both bloggers' and readers' activities in terms of the contributions that they make collaborative co-creative processes. Again, while beyond the scope of the current paper, we believe that such theorizing will be an important aspect of developing the field, not only of studying blogging, but of social media more generally.

\section{Future Work}

This paper has focused on the relationships between readers and bloggers in the context of political blogs, and how the interactions between them constitute a collaborative process of co-creation. These findings arise from close study of two political blogs, chosen in part for the blogger-reader interactions they evidence. Future work should examine whether these experiences of co-creation also occur among bloggers and readers of A-list blogs. Furthermore, the co-creative processes found in this study may be particular to political blogs. Therefore, it is important to examine interactions between readers and bloggers in the context of blogs that are 
not expressly political, such as technology blogs, food blogs, video game blogs, or knitting blogs. Such studies can help examine whether viewing blogging as collaborative co-creation is useful and informative in other contexts, or if the various motivations for participating in these different styles of blogs may lead to forms of blogger-reader interactions that can be better understood in different terms.

While blogging is a rich field site in which to explore such processes, it is certainly not the only possibility. Other social media, such as Flickr, Twitter, or YouTube, offer possibilities to explore which aspects of blog reading are specific to blogs and which are applicable to social media more generally. For example, in blogging, posts by bloggers and comments by readers are generally both textual, while in Flickr and YouTube, the photo or video artifacts around which interaction occurs are in a different medium than the textual comments. Thus, readership in these other social media may be accompanied by different modes of contribution than those presented here in the context of blogs. In the case of Twitter, there is no obvious analog to the post-comment structure of blogging; a user can reply to a tweet (a single message broadcast on Twitter), but that reply becomes an independent tweet itself rather than becoming associated only with the tweet to which it was a reply. When discussing promptness of comments above, we suggested that the ephemerality of blogs, despite being a written medium, may cause them to take on certain qualities of oral communication (cf. Ong 1980). Due to the highly conversational nature of Twitter, we expect that such orality would be even more conspicuous than in blogging. Finally, blogging is accomplished through a panoply of various tools, from "push-button blogging" sites such as Blogger (http://blogger.com), to blogging tools integrated into MySpace and Facebook, to server tools such as WordPress (http://wordpress.org) and Movable Type (http://movabletype.com), to custom content management systems designed using Ruby on Rails (http://rubyonrails.org) or Plone (http:// plone.org). While Technorati attempts to be a comprehensive index of blogs, there is no single provider of blogging. In contrast, Flickr and YouTube are services provided and owned by single companies, currently Yahoo and Google, respectively. On the one hand, such single-party ownership restricts technological features, e.g., YouTube users cannot create custom plugins for their channels. On the other hand, Twitter, also owned and operated by a single company (currently, Twitter, Inc.), can be accessed via the Twitter website or through a variety of third party clients and applications. Furthermore, many of these leading services have niche competitors, such as Picasa for photo-sharing and Jaiku for blogging. Blogging, Twitter, YouTube, Flickr, and others all have peculiarities to their social, technological, corporate, and organizational configurations. These complexities hint at the rich possibilities for studies of readers, reading, and readership in various forms of social media. Furthermore, if we recognize the process of creating social media as a collaborative one shared by authors and audience, some of the concepts used to describe those processes may be useful in understanding other types of collaborative work. 
One of the more provocative questions relevant specifically to political blogs is whether reading political blogs can serve as a form of political participation. McKenna and Pole (2004) argue that political bloggers see their blog as a form of political participation, while Wallsten (2005) suggests that political blogging should be considered not political participation but rather a form political expression. Farrell et al. (2008) find that reading political blogs is connected to increased political participation, in terms of voting, donating to candidates, and convincing others to vote for a candidate. Here, we have argued that reading political blogs itself constitutes a form of political participation, partly due to the ways in which the activities involved in reading a political blog can include debate, deliberation, and shifting of opinions, and partly due to the connection between reading blogs and discussions that happen outside of the blog. Further research should explore more fully what kind of political participation blog reading might constitute, and, moreover, if it might be possible to determine whether this form of participation is overall beneficial or detrimental. For example, our data suggest that people are reading blogs not as an alternative to other forms of participation (e.g., attending rallies or demonstrations), but rather as an alternative to not participating at all. In that sense, blog reading might be seen in a positive light. However, many have argued that blogs and other online media more often serve to reinforce previously held opinions than to expose us to a variety of alternative opinions (e.g., Farrell et al. 2008; Garrett 2005), casting them in a somewhat more negative light. Future work should seek to understand both the benefits and detriments of engaging in these novel, technologicallymediated form of political participation.

Such research should also carefully consider the relationships between political participation and political ideology. The study presented here intentionally included readers from one liberal blog and one conservation blog. However, due to the small study size, as well as technical differences between the two blogs, it is difficult to make conclusive comparisons. Furthermore, during our analysis, ideology became an important factor for all participants, less in terms of how their ideology influenced their reading practices and more in terms of the nuanced and complex ways they identified their own political ideologies. While studies could be done comparing reading and participation between conservative and liberal blogs, we suggest that a more fruitful area of study is in work that moves beyond traditional left/right binarisms, instead understanding the more fluid and subtle ways in which blog readers constitute their ideologies.

In terms of connecting to previous research on lurkers, it may be informative to study those blog readers who do not explicitly interact through comments, email, or any other means. While there were some such readers in this study, they were not a central focus. Future work should explore whether those "lurking" blog readers have the same feeling of "being a part" of the blogs they read, as well as whether and how such readers contribute to the collaborative process of cocreating the blog. 
While previous research suggests that people strongly prefer blogs (Farrell and Drezner 2008) and news sources in general (Garrett 2005) that align with their own opinions, the findings presented here indicate that it is common for readers occasionally to read blogs whose political ideology differs from their own. Exposure to differing opinions has the potential to greatly impact political deliberation and individual decision making. Thus, the relationship between reading political blogs and exposure to varying opinions is an important issue to address. However, doing so can be quite difficult, as studies generally rely on self-report or a simulated laboratory environment to collect data. One possibility may be using automatic internet traffic loggers to determine subjects' actual patterns of internet use. Not only does this pose obvious privacy concerns that have confounded previous attempts to $\log$ blog reading behavior (Baumer et al. 2008), installing a logger to track internet usage may actually cause individuals to change internet usage itself. On the one hand, such an impact might negatively affect the data gathered so as to make them not as informative about actual reading patterns. On the other, combining such an approach with qualitative methods could elicit informative reflections from participants as to if, how, and why they altered their reading practices and patterns when they knew they were being logged.

One of the motivating factors in choosing political blogs as a field site was to understand the relationship between online interactions through blogs and offline political events and activities. However, during the course of our analysis, it became clear that the analytic distinction between online and offline not only lacked utility, in that it did not align with readers' experiences, but it also obscured the ways in which blogs were made meaningful by readers. As previously noted, online and offline are not mutually exclusive (Miller and Slater 2000), but they are not completely coincident, either. We suggest that this work problematizes the online/offline distinction, and that future work can help explore alternative conceptualizations, e.g., blogs as a medium for social interaction (Kato 2006; boyd 2006) or blogs as but one component of a communicative ecology (Foth and Hearn 2007; Tacchi et al. 2003).

The results presented here were informed by qualitative data gathered over a period of approximately two and a half months. While this was a long enough time period to become familiar with the readers and bloggers who participated in our study, it was not long enough to see significant changes in their interactions. Baumer et al. (2008) point out that reader-blogger interactions are dynamic, developing, changing, and evolving over time. Paul describes how the relationships between his blogging and his own reading habits changed over the years from when he first started blogging. Future studies should be conducted on a longer time scale in order to examine the process of how these interactions change and evolve over time. Large-scale, quantitative studies should also be carried out to help determine what aspects of such interactions are common among which blogging populations. 
One last important area for future work is the development of computational tools aimed specifically at blog readers. While there has been a great deal of work done on tools aimed at writing blogs, such as Blogger, Live Journal, Xanga, etc., there has been little focus on tools designed specifically to support reading practices. This study did not focus on technology design and thus does not aim to provide substantial implications for design (cf. Dourish 2006), although we have described above some potential "inspirations for design" (cf. Terrenghi et al. 2006) of tools for readers of political blogs. Beyond those ideas, there are two important points to be made with respect to the design of such tools. First, we wish to take this opportunity to draw the research community's attention to tools for blog reading as an interesting and important research and design space. While there has been some work in this area, e.g., (Gamon et al. 2008; Baumer et al. 2010; Baumer and Fisher 2008; Dennis and Jarrett 2005), very little has focused specifically on supporting the activity of reading blogs. Second, we suggest that any successful tool for blog readers must support, if not facilitate, the various roles that readers play in shaping a blog. This paper describes the collaborative process of co-creation shared in by bloggers and readers; tools for blog reading should be designed around that process.

\section{Conclusion}

Blogging is not only a social activity (Nardi, Schiano \& Gumbrecht 2004), it is also a collaborative activity. The findings presented have explored various ways in which bloggers and readers are collaboratively involved in the process of co-creating a blog. The study described here is the first, to the authors' knowledge, that examines the same blogging activity through the experiences of both bloggers and readers. This analysis allows us to see how a blog is created not only through the process of writing or the process of reading, but also through the interactions of people engaging in those processes. While some activities are more reading oriented, such as commenting, and some activities are more writing oriented, such as posting, bloggers and readers both play different but vital roles in co-creating the blog.

The question then becomes how to study such distributed activities in the creation of social media. Previous research on blog readers (Baumer et al. 2008) emphasized the important of interaction, that a blog should be defined in terms of the types of interactions it allows, invites, or compels. Here, we claim that if blogging is the process of creating a blog, and if a blog is created through the interactions among bloggers and readers, then not only is the interaction important, but moreover, the interaction itself is what constitutes blogging. Blogging is in part the process of creating a blog, but that creation process is a collaborative one in which both bloggers and readers take part. This collaboration is realized through a complex set of interactions, described in the findings presented here in terms of several salient issues: readers' participation in the blog and the various ways in which that participation is constituted; the 
interconnections and mutual influences between reading, political ideology, and political participation; and impacts of the differences and similarities between blogs and mainstream media on readers' interactions with the blog and the blogger. We argue that these examples demonstrate the ways in which bloggers and readers interact. It is through those interactions that a blog is collaboratively co-created, and it is that collaborative process of cocreation that makes the blog a blog. If we are to understand blogs or any other social media, we must focus not on the media being produced, not on the artifactual byproducts of this interaction, but on the interaction itself, on the socializing in social media.

\section{Acknowledgments}

We are highly grateful to our participants for investing their time and effort in our research. We thank Rodrigo Lois for helping with some of the data collection for this study; R. Kelly Garrett, Andrea Forte, and Kevin Wallsten, for comments on earlier drafts of this paper; and the anonymous JCSCW reviews for their helpful comments, critiques, and suggestions. We also thank the Donald Bren School of Information and Computer Sciences, the California Institute for Telecommunications and Information Technology, and the Sloan Foundation for their support of this research.

Open Access This article is distributed under the terms of the Creative Commons Attribution Noncommercial License which permits any noncommercial use, distribution, and reproduction in any medium, provided the original author(s) and source are credited.

\section{Notes}

1. Though some single-author blogs have occasional guest-bloggers, these blogs are still strongly associated with the primary blogger.

2. http://technorati.com/blogs/directory/politics/

3. http://tamsys.sourceforge.net/

\section{References}

Adamic, L. A., \& Glance, N. (2005). The Political Blogosphere and the 2004 U.S. Election: Divided They Blog. In Weblogging Ecosystem Workshop. Japan.

Baumer, E., \& Fisher, D. (2008). Smarter blogroll: An exploration of social topic extraction for manageable blogrolls. In Hawai'i Int'l Conf on Sys Sci (HICSS). Waikoloa Village, HI: IEEE Computer Society, p. 155.

Baumer, E., Sueyoshi, M., \& Tomlinson, B. (2008). Exploring the role of the reader in the activity of blogging. In ACM Conference on Human Factors in Computing Systems (CHI 2008). Florence, Italy: ACM, pp. 1111-1120.

Baumer, E. P. S., Sinclair, J., \& Tomlinson, B. (2010). “America Is Like Metamucil:” Critical and creative thinking about metaphor in political blogs. In ACM Conference On Human Factors In Computing Systems (CHI 2010). Atlanta, GA: ACM Press, pp. 1437-1446. 
Blood, R. (2004). How blogging software reshapes the online community. Communications of the $A C M, 47(12), 53-55$.

Boyd, D. (2002). Faceted identity: Managing representation in a digital world. Master's Thesis. Massachusetts Institute of Technology, Media Arts and Sciences.

Boyd, D. (2006). A blogger's blog: Exploring the definition of a medium. Reconstruction: Studies in Contemporary Culture, 6(4).

Carpenter, F., et al. (2004). Methods of facilitating reforestation of tropical degraded land with the native timber tree, Terminalia amazonia. Forest Ecology and Management, 202, 281-291.

Cooley, C. H. (1902). Human nature and the social order. New York: Charles Scribner's Sons.

Dennis, B., \& Jarrett, A. (2005). NusEye: Visualizing network structure to support navigation of aggregated content. In HI Int'l Conf Sys Sci (HICSS). Big Island, HI: IEEE Comp Soc.

Dourish, P. (2006). Implications for design. In ACM Conference on Human Factors in Computing Systems (CHI 2006). Montréal, Québec, Canada: ACM Press, pp. 541-550.

Drezner, D. W., \& Farrell, H. (2004). The power and politics of blogs. In American Political Science Association's Annual Meeting.

Farrell, H., \& Drezner, D. W. (2008). The power and politics of blogs. Public Choice, 134(1-2), $15-30$.

Farrell, H., Lawrence, E., \& Sides, J. (2008). Self-segregation or deliberation? Blog Readership, Participation and Polarization in American Politics. In MPSA Annual Nat'l Conf. Chicago. Available at: SSRN eLibrary, http://papers.ssrn.com/sol3/papers.cfm?abstract_id=1151490 [Accessed July 8, 2008].

Foot, K., \& Schneider, S. M. (2006). Web campaigning, The MIT Press.

Foth, M., \& Hearn, G. (2007). Networked individualism of urban residents: Discovering the communicative ecology in inner-city apartment buildings. Information, Communication \& Society, 10(5), 749-772.

Galegher, J., Sproull, L., \& Kiesler, S. (1998). Legitimacy, authority, and community in electronic support groups. Written Communication, 15, 493-530.

Gamon, M., et al. (2008). BLEWS: Using blogs to provide context for news articles. In Int'l Conf on Weblogs and Social Media. Seattle, WA: AAAI Press.

Garrett, R. K. (2005). Exposure to controversy in an information society. Ph. D. Dissertation. University of Michigan, School of Information.

Garrett, R. K. (2006). Protest in an information society: A review of literature on social movements and new ICTs. Information, Communication \& Society, 9(2), 202.

Gilbert, E., Bergstrom, T., \& Karahalios, K. (2009). Blogs are echo chambers: blogs are echo chambers. In HI Int'l Conf SysSci (HICSS). Big Island, HI: IEEE Computer Society, pp. 1-10.

Gumbrecht, M. (2004). Blogs as "Protected Space". In Workshop on the weblogging ecosstem: aggregation, analysis, and dynamics. New York: ACM Press.

Habermas, J. (1991). The structural transformation of the public sphere: An inquiry into a category of bourguis society. Cambridge: MIT.

Hong, L., et al. (2010). FeedWinnower: Layering structures over collections of information streams. In ACM Conference on Human Factors in Computing Systems (CHI 2010). Atlanta, GA: ACM Press.

Kato, H. (2006). Japanese Youth and Imagining of Keitai. In M. Ito, D. Okabe, \& M. Matsuda (Eds.), Personal, portable, pedestrian: Mobile phones in Japanese life (pp. 103-119). Cambridge: MIT.

Kim, J., Wyatt, R. O., \& Katz, E. (1999). News, talk, opinion, participation: the part played by conversation in deliberative democracy. Political Communication, 16, 361-385.

Lave, J., \& Wenger, E. (1991). Situated learning: Legitimate peripheral participation. Cambridge University Press.

Lee, Y., Chen, F., \& Jiang, H. (2006). Lurking as participation: A community perspective on lurkers' identity and negotiability. In Proceedings of the 7th international conference on 
Learning sciences. Bloomington, Indiana: International Society of the Learning Sciences, pp. 404-410. Available at: http://portal.acm.org/citation.cfm?id=1150034.1150093 [Accessed March 19, 2010].

Lenhart, A., 2005. Unstable texts: An ethnographic look at how bloggers and their audience negotiate self-presentation, authenticiy, and norm formation. Master's Thesis. Georgetown University, Graduate School of Arts and Sciences.

Lofland, J., \& Lofland, L. (1995). Analyzing social settings: A guide to qualitative observation and analysis. Belmont: Wadsworth.

Losse, K. (2008). Political views. The Facebook Blog. Available at: http://blog.facebook.com/blog. php?post $=10499722130$ [Accessed July 10, 2008].

McKenna, L., \& Pole, A. (2004). Do blogs matter? Weblogs in American Politics. In Amer Poli Sci Assoc's Annual Mtg. Chicago, IL.

Miller, D., \& Slater, D. (2000). Chapter one-conclusions. In The internet: An ethnographic approach. Oxford: Berg.

Nardi, B. A., Schiano, D., \& Gumbrecht, M. (2004). Blogging as social activity, or, would you let 900 million people read your diary? In ACM Conference on Computer-Supported Cooperative Work (CSCW 2004). Chicago, Illinois: ACM Press, pp. 222-231.

Nardi, B. A., Schiano, D., Gumbrecht, M., \& Swartz, L. (2004). Why we blog. Communications of the Association for Computing Machinery, December, 41-46.

Nonnecke, B., \& Preece, J. (2001). Why Lurkers Lurk. In Americas Conference on Information Systems (AMCIS). Boston, p. Paper 294.

Ong, W. (1980). Orality and literacy (2003 ed.). New York: Routledge.

Preece, J., \& Shneiderman, B. (2009). The reader-to-leader framework: motivating technologymediated social participation. AIS Transactions on Human-Computer Interaction, 1(1), 13-32.

Preece, J., Nonnecke, B., \& Andrews, D. (2004). The top five reasons for lurking: improving community experiences for everyone. Computers in Human Behavior, 20(2), 201-223.

Reed, A. (2005). 'My blog is me': texts and person in UK online journal culture (and anthropology). Ethnos: Journal of Anthropology, 70(2).

Rosenstone, S. J., \& Hansen, J. M. (1993). Mobilization, participation, and democracy in America. New York: Macmillian.

Schiano, D., et al. (2004). Blogging by the rest of us. In CHI Extended Abstraction on Human Factors in Computing Systems. Vienna, Austria: ACM Press, pp. 1143-1146.

Schmidt, J. (2007). Blogging practices: an analytical framework. Journal of Computer-Mediated Communication, 12(4).

Tacchi, J., Slater, D., \& Hearn, G. (2003). Ethnographic action research: A user's handbook. New Dehli: UNESCO.

Terrenghi, L., Harper, R., \& Sellen, A. (2006). Inspirations for design. In Open Session, Conference on Ubiquitous Computing. Orange County, CA.

Voida, A., et al. (2008). Asymmetry in media spaces. In ACM Conference on Computer-Supported Cooperative Work (CSCW 2008). San Diego, CA, USA: ACM, pp. 313-322.

Walker, D. M. (2006). Blog commenting: a new political information space. In American Society for Information Science and Technology. Austin, TX.

Wallsten, K. (2005). Political blogs and the bloggers who blog them: Is the political blogosphere and echo chamber. In Amer Poli Sci Assoc's Annual Mtg. Washington, D.C.

Wenger, E. (1998). Communities of practice, Cambridge University Press. 